\title{
Integrative omics reveals subtle molecular perturbations following ischemic conditioning in a porcine kidney transplant model
}

\author{
Darragh P. O'Brien ${ }^{1 *+} @$, Adam M. Thorne ${ }^{1,2+}$, Honglei Huang ${ }^{1,2}$, Elisa Pappalardo ${ }^{3}$, Xuan Yao ${ }^{1}$, \\ Peter Søndergaard Thyrrestrup ${ }^{4,5}$, Kristian Ravlo $^{4,6}$, Niels Secher ${ }^{6,7}$, Rikke Norregaard ${ }^{4,6}$, Rutger J. Ploeg ${ }^{2 *}$, \\ Bente Jespersen ${ }^{4,6^{*}}$ and Benedikt M. Kessler ${ }^{1 *}$ (])
}

\begin{abstract}
Background: Remote Ischemic Conditioning (RIC) has been proposed as a therapeutic intervention to circumvent the ischemia/reperfusion injury (IRI) that is inherent to organ transplantation. Using a porcine kidney transplant model, we aimed to decipher the subclinical molecular effects of a RIC regime, compared to non-RIC controls.

Methods: Kidney pairs $(n=8+8)$ were extracted from brain dead donor pigs and transplanted in juvenile recipient pigs following a period of cold ischemia. One of the two kidney recipients in each pair was subjected to RIC prior to kidney graft reperfusion, while the other served as non-RIC control. We designed an integrative Omics strategy combining transcriptomics, proteomics, and phosphoproteomics to deduce molecular signatures in kidney tissue that could be attributed to RIC.
\end{abstract}

Results: In kidney grafts taken out $10 \mathrm{~h}$ after transplantation we detected minimal molecular perturbations following RIC compared to non-RIC at the transcriptome level, which was mirrored at the proteome level. In particular, we noted that RIC resulted in suppression of tissue inflammatory profiles. Furthermore, an accumulation of muscle extracellular matrix assembly proteins in kidney tissues was detected at the protein level, which may be in response to muscle tissue damage and/or fibrosis. However, the majority of these protein changes did not reach significance $(p<0.05)$.

Conclusions: Our data identifies subtle molecular phenotypes in porcine kidneys following RIC, and this knowledge could potentially aid optimization of remote ischemic conditioning protocols in renal transplantation.

Keywords: Remote ischemic conditioning, Kidney transplantation, Proteomics, Transcriptomics, Integrative omics, Inflammation

\footnotetext{
*Correspondence: darragh.obrien@ndm.ox.ac.uk; rutger.ploeg@nds.ox.ac.uk; bente.jespersen@clin.au.dk; benedikt.kessler@ndm.ox.ac.uk

${ }^{\dagger}$ Darragh P. O'Brien and Adam M. Thorne contributed equally to the work

${ }^{1}$ Target Discovery Institute, Centre for Medicines Discovery, Nuffield Department of Medicine, University of Oxford, Oxford, UK

${ }^{2}$ Nuffield Department of Surgical Sciences and Oxford Biomedical Research Centre, University of Oxford, Oxford, UK

${ }^{4}$ Department of Renal Medicine, Aarhus University Hospital, Aarhus, Denmark

Full list of author information is available at the end of the article
}

\begin{abstract}
Background
Organ donation and transplantation inevitably involve periods of both warm and cold ischemia, due to diminished or restricted blood flow, which deprives the donor organ tissue of the oxygen required to maintain cellular metabolism. Subsequent reperfusion at time of implantation of the ischemic graft results in tissue damage including several molecular hallmarks, such as hypoxic stress and production of reactive oxygen species, a rapid reduction of ATP levels, intracellular acidosis, and diminished
\end{abstract}

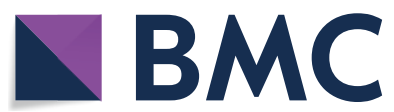

(c) The Author(s) 2022. Open Access This article is licensed under a Creative Commons Attribution 4.0 International License, which permits use, sharing, adaptation, distribution and reproduction in any medium or format, as long as you give appropriate credit to the original author(s) and the source, provide a link to the Creative Commons licence, and indicate if changes were made. The images or other third party material in this article are included in the article's Creative Commons licence, unless indicated otherwise in a credit line to the material. If material is not included in the article's Creative Commons licence and your intended use is not permitted by statutory regulation or exceeds the permitted use, you will need to obtain permission directly from the copyright holder. To view a copy of this licence, visit http://creativecommons.org/licenses/by/4.0/. The Creative Commons Public Domain Dedication waiver (http://creativeco mmons.org/publicdomain/zero/1.0/) applies to the data made available in this article, unless otherwise stated in a credit line to the data. 
nutritional supplies [1]. Combined, these insults lead to the activation of apoptotic and necrotic pathways, and if uncorrected, to organ dysfunction, rejection, and eventually graft fibrosis [2]. Acute kidney injury (AKI) induced by ischemia and subsequent reperfusion is called delayed graft function in the clinical transplant setting and leads to the need for dialysis, as well as a risk of poorer transplant outcome [3]. Remote ischemic conditioning (RIC) has been proposed as a therapeutic modality to mitigate the effects of ischemia/reperfusion injury (IRI) in organ transplantation $[4,5]$. RIC is a simple procedure which creates short and repetitive cycles of non-damaging ischemia, usually applied to a leg or arm, which may reduce damaging effects caused by IRI in the target organ. RIC has shown various outcomes in pre-clinical studies in both kidney and other organs such as lung, liver, small intestine and in a variety of situations and species [6-12]. Several pre-clinical studies have demonstrated a reduction in IRI and improved early graft function in the recipient [13-16]. Regarding liver or kidney transplantation and AKI, a protective effect of RIC in rodent renal models has been described, however the transition into the human setting has been challenging [17]. Our previous work in a porcine model of kidney transplantation suggested a beneficial effect of RIC on early graft perfusion and function evidenced by a significantly improved renal plasma perfusion and glomerular filtration rate (GFR) [18]. However, when RIC was applied to kidney transplant patients in a clinical trial (CONTEXT), no clinical benefit was observed, although the trial did reveal distinct subclinical effects on kidney transplants at the molecular level $[17,19,20]$. We therefore revisited our RIC study carried out in pigs which showed potential positive effects of RIC, and which had stimulated us to design the clinical CONTEXT study. Using samples obtained from our original porcine RIC model [18], we have now studied whether we could detect any proteomic and transcriptomic changes in recipient tissue using advanced quantitative mass spectrometry and RNA sequencing. In this study, we describe changes in molecular profiles comparable to the ones observed in human kidneys subjected to RIC.

\section{Methods}

\section{Transplant model}

The effects of RIC versus non-RIC controls in porcine kidney transplants were evaluated in a paired randomized study, the design of which was described in detail by Soendergaard et al. [18]. Briefly, eight kidney pairs were taken from eight brain-dead donor pigs (60$64 \mathrm{~kg}$ ) and transplanted into sixteen bilateral nephrectomized recipient pigs (14-16 kg), which were randomly given RIC or non-RIC within each pair. Prior to organ reperfusion, RIC was performed as indicated below. Pigs were surveyed, under anesthesia, for $10 \mathrm{~h}$ after transplantation, assessing renal function by GFR, renal plasma perfusion and known renal biomarkers. $\mathrm{A} 10 \mathrm{~h}$ was the maximal time the animals could be kept stable on anesthesia. Biopsies were not taken during the observation time to minimize bleeding risk. Nephrectomy of the recipients occurred just before implantation of the graft. To assess GFR over time, we measured urinary clearance of a filtered isotope, a method that is able to detect minor changes of GFR. The study was approved by the National Committee on Animal Research Ethics no. 2008-5611584 (Animal Experiments Inspectorate, Copenhagen, Denmark) and conducted in accordance with the "Principles of Laboratory Animal Care" (NIH publication Vol. 25, No. 28 revised 1996).

\section{Sample retrieval and ischemia regimen}

Kidney retrieval, transplantation and RIC was performed by experienced transplant surgeons as described previously (Fig. 1) [18]. Briefly, female Danish Landrace pigs were anaesthetized and placed under mechanical ventilation. Female pigs were selected for two reasons: firstly, they are generally easier to house and keep than their male counterparts, and secondly, it can be challenging to perform urinary catherization on male pigs. Ringer's acetate was infused to maintain fluid balance and arterial pressure. Brain death was induced by increasing intracranial pressure using the method described by Barklin and colleagues [21]. Briefly, two holes were drilled into the cranium approximately $15 \mathrm{~mm}$ posterior and anterior to the coronal suture on opposing sides, and approximately $10 \mathrm{~mm}$ lateral to the sagittal suture. Into the anterior cavity, a 20 Fr Foley catheter was inserted and a catheter balloon implanted into the epidural space. To record continual intracranial pressure (ICP) measurements, a steel introducer needle from a 22G Venflon ${ }^{\circledR}$ (Becton Dickinson, Franklin Lakes, NJ, USA) was inserted subdurally into the posterior cavity. Brain death was realized when intracranial pressure exceeded systolic blood pressure. Kidneys were extracted, perfused and stored in $5{ }^{\circ} \mathrm{C}$ cold storage solution. Two recipients were transplanted simultaneously after having their native kidneys removed. A RIC regimen was undertaken whereby the abdominal aorta was clamped above the aortic bifurcation but below the renal arteries in four cycles, with each cycle consisting of $5 \mathrm{~min}$ of ischemia and $5 \mathrm{~min}$ of reperfusion. Surgeons and investigators were unblinded to the treatment during the experiments. Renal samples from kidneys in the RIC and non-RIC groups were taken prior to termination after $10 \mathrm{~h}$ reperfusion and snap frozen in liquid nitrogen for transcriptomic and proteomic analysis. 


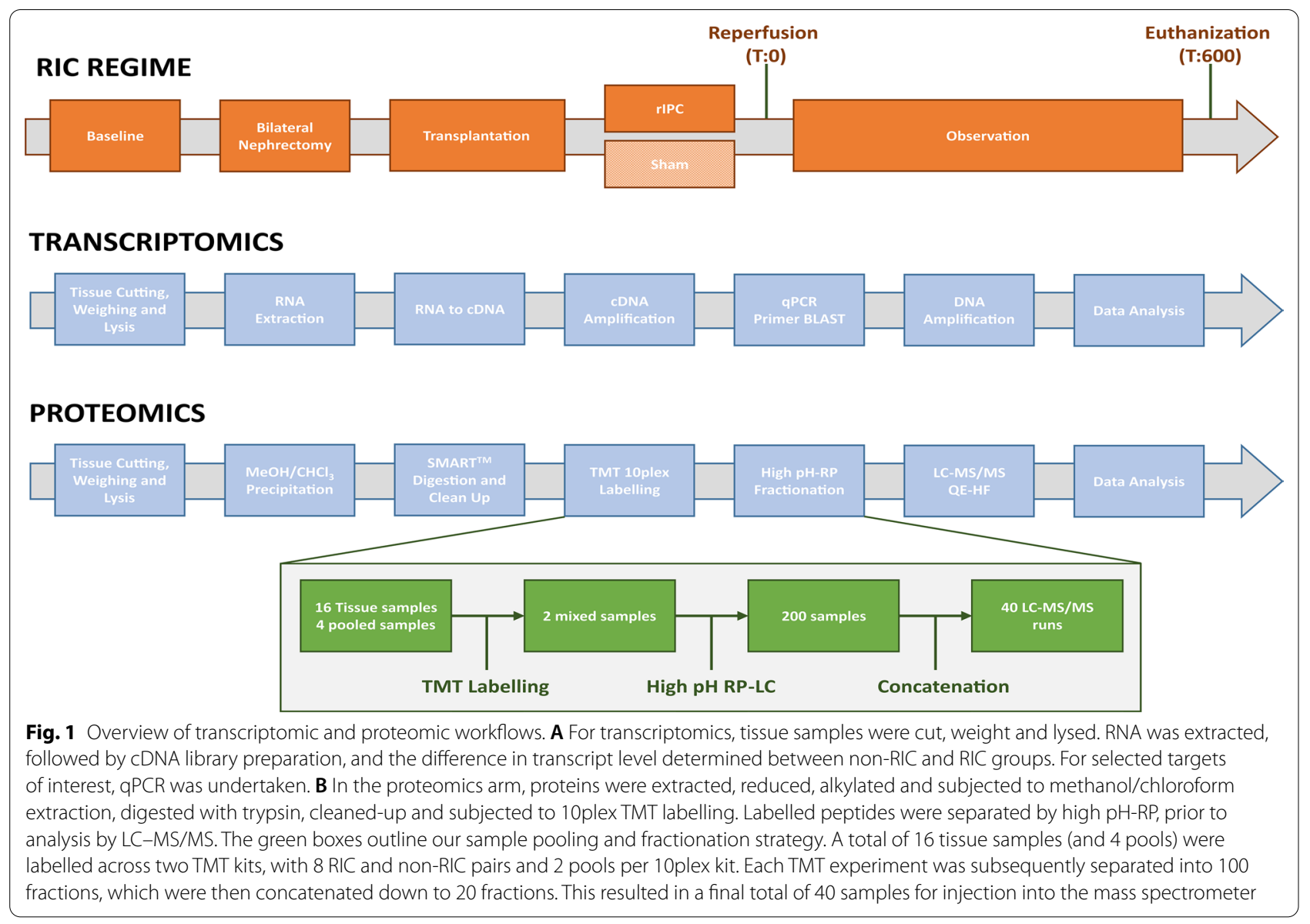

\section{Transcriptomics analysis \\ Sample preparation}

Sixteen tissue sections from the kidney medulla and cortex (weighing approximately $20 \mathrm{mg}$ ) were lysed using a Precellys 24 homogenizer (Bertin Instruments) in $350 \mu \mathrm{L}$ of RNeasy Mini kit RLT lysis buffer. RNA was then extracted using RNeasy Mini kit (Qiagen, cat number: 74104) as per manufacturer's instructions. Purified RNA was eluted in $40 \mu \mathrm{L}$ RNase-free $\mathrm{H}_{2} \mathrm{O}$ and the approximate concentration evaluated using a NanoDrop ND-1000 spectrophotometer. Approximately, $450 \mathrm{ng}$ of purified RNA was converted to cDNA by using highcapacity reverse transcription kit (Applied Biosystems, cat number: 4368814). For each reaction, a mix of $2 \mu \mathrm{L}$ $10 \times$ RT buffer, $0.8 \mu \mathrm{L} 25 \times$ dNPT mix, $2 \mu \mathrm{L} 10 \times$ RT random primers, $1 \mu \mathrm{L}$ Multiscribe reverse transcriptase, $3.2 \mu \mathrm{L}$ nuclease free $\mathrm{H}_{2} \mathrm{O}$ and $11 \mu \mathrm{L}$ of RNA $(40.9 \mathrm{ng} / \mu \mathrm{L})$ was prepared. This was then primed at $25^{\circ} \mathrm{C}$ for $10 \mathrm{~min}$, incubated at $37^{\circ} \mathrm{C}$ for $120 \mathrm{~min}$ and inactivated at $85^{\circ} \mathrm{C}$ for $5 \mathrm{~min}$ in a thermal cycler (VWR UNO 96). cDNA was then stored at $-20{ }^{\circ} \mathrm{C}$ until further use. Transcriptomics analysis was performed on $\sim 50 \mathrm{fmol}$ of the sample library and sequenced on a NextSeq 500 sequencer (Illumina) achieving, $\sim 30$ million reads in total (Additional file 2: Table S1). An overview of the transcriptomics workflow is given in Fig. 1.

For transcriptomics data analysis, FASTQ files were converted to Binary-sequence Alignment Format (BAM) files using HISAT2 (v2.1.0) and Samtools (v1.3). The DESeq2 $\mathrm{R}$ package (Bioconductor) was used for normalization, visualization, and differential analysis of the data. Furthermore, BAM files were also imported into Perseus software (v1.6.0.2) for comparative analysis, and genome annotation was performed using the Sus scrofa FASTA cDNA database (http://www.ensembl.org). Reads per kilo per million (RPKM) values were calculated by a normalization step dividing by the sum (Normalization $\rightarrow$ Divide), followed by dividing normalized values by gene length, multiplying by 109 , and taking the $\log _{2}$ values. The transcriptomics dataset was deposited in ArrayExpress (AE) at EBI under the accession number E-MTAB-10872 [22].

\section{$q-P C R$}

For q-PCR, primers for selected genes of interest were designed using Primer BLAST (see Table 1 for 
Table 1 List of gene names and primer sequences used to conduct qPCR

\begin{tabular}{llll}
\hline Oligo name & Forward sequence $\left(\mathbf{5}^{\prime} \mathbf{- \mathbf { 3 } ^ { \prime } )}\right.$ & Reverse sequence $\left.\mathbf{( 5}^{\prime} \mathbf{- 3}^{\prime}\right)$ & Scale $(\boldsymbol{\mu m o l e})$ \\
\hline PDZD3 & CCCTGTAAGTGCCCTGCTAT & CTCCAGGGATCAGAAGGATCG & $0.025 \mu$ mole \\
LTB4R & TGGTACTTCCTCTCTGGCTGA & CCATTGCAAGGACAGGCTTT & $0.025 \mu$ mole \\
RASL10A & TTGCATTTGGGTAAACCTGGAA & GCCACTCCTCCAAGCTTAATTC & $0.025 \mu$ mole \\
SLC16A3 & AGGTAACCTGAGACCTGGCT & TGGTTCCGCGTCCCTGG & $0.025 \mu$ mole \\
IL1B & CCAATTCAGGGACCCTACCC & GTTTTGGGTGCAGCACTTCAT & $0.025 \mu$ mole \\
Beta actin & TGTCATGGACTCTGGGGATG & GGGCAGCTCGTAGCTCTTCT & $0.025 \mu$ mole \\
\hline
\end{tabular}

A total of five genes were selected for qPCR validation of transcriptomics results, with beta actin serving as a control

sequences), parameters included spanning exon-exon junctions, a target size of $200 \mathrm{bp}$ and a maximum product size of 150. A beta-actin control gene was used as a reference [23]. A total of $20 \mu \mathrm{g}$ of cDNA was used for each $20 \mu \mathrm{L}$ reaction, in combination with $200 \mathrm{nM}$ of each forward and reverse primer (Invitrogen) and Fast SYBR Green Master Mix (Applied Biosystems, cat number: 4385612), according to the manufacturer's instructions. The reaction plates were run on a Roche Lightcycler 480 , with an initial pre-incubation of $37^{\circ} \mathrm{C}$ for $30 \mathrm{~s}$, then denaturing at $95^{\circ} \mathrm{C}$ for $3 \mathrm{~s}$, followed by annealing at $60^{\circ} \mathrm{C}$ for $30 \mathrm{~s}$. Denaturing and annealing was repeated for 40 runs before conducting melt curve analysis. Using Roche Lightcycler analysis software, the noise band (baseline) was altered to exclude any background noise whilst staying within the lower third of the linear section of the curve to gain initial $\mathrm{Ct}$ values [24]. Average $\mathrm{Ct}$ values for beta-actin were then subtracted from average $\mathrm{Ct}$ values of target genes to get dCt. RIC vs non-RIC groups and different time points were then compared using $2^{\wedge}-(x-y)$ to obtain relative expression levels and plotted on a boxdot graph.

\section{Proteomics analysis Sample preparation}

In accordance with the transcriptomics study, sixteen tissue samples (weighing approximately $20 \mathrm{mg}$ ) were lysed using a Precellys 24 homogenizer (Bertin Instruments) in $30 \mu \mathrm{L}$ RIPA and $6 \mathrm{M}$ urea lysis buffer per $1 \mathrm{mg}$ of tissue. Samples were reduced with $200 \mathrm{mM}$ tris(2-carboxyethyl)phosphine (TCEP) at $55{ }^{\circ} \mathrm{C}$ for $1 \mathrm{~h}$ and alkylated with $375 \mathrm{mM}$ iodoacetamide (IAA) for $30 \mathrm{~min}$ at room temperature. Following this, protein precipitation via methanol/chloroform precipitation was performed. The resulting pellet was dried and resuspended in $50 \mu \mathrm{L}$ triethylammonium bicarbonate (TEAB) and the protein concentration determined using a BCA assay (Pierce). In total, $100 \mu \mathrm{g}$ of protein was digested by trypsin using the SMART method (Thermo Scientific). In brief, each sample was loaded into a SMART digestion tube (Thermo Fisher Scientific, Cat no 60109-103) containing $150 \mu \mathrm{L}$ of SMART digestion buffer. These were then incubated at $70{ }^{\circ} \mathrm{C}$ at $1400 \mathrm{rpm}$ for $2 \mathrm{~h}$ on a heat shaker (Eppendorf) and spun at $2500 \times g$ for $5 \mathrm{~min}$, with collection of the supernatant. Samples were de-salted using SOLA $\mu$ SPE plates (Thermo Scientific, Cat no. 60109-103). Columns were equilibrated using $100 \%$ acetonitrile (ACN), then $0.1 \%$ trifluoroacetic acid (TFA). The samples were then diluted with 1\% TFA and pulled through the column using a vacuum pump. They were then washed with $0.01 \%$ TFA and eluted in $100 \mu \mathrm{L} 65 \%$ acetonitrile. Eluates were dried using a vacuum concentrator (SpeedVac, Thermo Scientific) and resuspended in $100 \mu \mathrm{L}$ of $100 \mathrm{mM}$ TEAB for Tandem Mass Tag (TMT) labelling.

\section{TMT labelling and high $\mathrm{pH}$ fractionation}

TMT 10plex reaction groups were used to label the digested peptides. Groups comprised of eight samples (four sets of RIC and non-RIC) and two pools; one undiluted, and one diluted 1:5, with the pools allowing us to assess the dynamic range of the measurement. For labelling, $41 \mu \mathrm{L}$ of TMT label was added to each sample and incubated for $1 \mathrm{~h}$ at room temperature. To quench the reaction, $8 \mu \mathrm{L}$ of $5 \%$ hydroxylamine was added to each sample and incubated at room temperature for $15 \mathrm{~min}$. Equal volumes of each RIC and non-RIC sample were then pooled into their respective groups to generate a RIC and non-RIC "pooled" sample, and de-salted using Sep-Pak C18 columns. The resulting eluent was dried using a vacuum concentrator (SpeedVac, Thermo Scientific) and resuspended in $120 \mu \mathrm{L}$ of buffer A (98\% MilliQ- $\mathrm{H} 2 \mathrm{O}, 2 \%$ acetonitrile, $0.1 \%$ TFA). The two combined TMT 10plex experiments were then pre-fractionated using high $\mathrm{pH} 10.0$ reversed-phase liquid chromatography (HPLC) into a total of 100 fractions for each. Fractions were subsequently concatenated into a total of 20 samples per TMT 10plex experiment and submitted for LC-MS/MS analysis. The proteomic sample preparation strategy is summarized in Fig. 1.

\section{Phosphoproteomics}

For phosphopeptide enrichment, $50 \mu \mathrm{g}$ of protein was removed from each of the 16 tissue samples and pooled into their respective RIC and non-RIC groups. Samples 
were reduced, alkylated, digested with trypsin, and desalted as above. Peptides $(\sim 400 \mu \mathrm{g})$ were eluted from Sep-Pak cartridges in $500 \mu \mathrm{L}$ buffer B (65\% ACN, 35\% MilliQ-H2O, and 0.1\% TFA). Phosphopeptide enrichment was performed using an Immobilized Metal Affinity Chromatography (IMAC) column with the aid of a Bravo Automated Liquid Handling Platform (Agilent) according to manufacturer's instructions.

\section{Mass spectrometry analysis}

Liquid chromatography tandem mass spectrometry (LC-MS/MS) analysis was undertaken using a Dionex Ultimate 3000 nano-ultra reversed-phase HPLC system with on-line coupling to a Q Exactive High Field (HF) mass spectrometer (Thermo Scientific). Samples were separated on an EASY-Spray PepMap RSLC C18 column (500 $\mathrm{mm} \times 75 \mu \mathrm{m}, 2 \mu \mathrm{m}$ particle size; Thermo Fisher Scientific) over a 60 min gradient of $2-35 \%$ ACN in $5 \%$ dimethyl sulfoxide (DMSO), 0.1\% FA, and the flow rate was $\sim 250 \mathrm{~nL} / \mathrm{min}$. The mass spectrometer was operated in data-dependent analysis mode for automated switching between MS and MS/MS acquisition. Full MS survey scans were acquired from 400 to $2000 \mathrm{~m} / \mathrm{z}$ at a resolution of 60,000 at $200 \mathrm{~m} / z$ and the top 12 most abundant precursor ions were selected for high collision energy dissociation fragmentation. MS2 fragment ion resolution was set to 15,000 .

\section{Data analysis}

MS raw data files were searched using Proteome Discoverer (v2.3; total proteome) and MaxQuant (v1.5.14; phosphoproteome) software packages. Search parameters included the allowance of two missed trypsin cleavages. Carbamidomethylation $(\mathrm{C})$ was set as a fixed modification, while TMT6plex (N-term, K), oxidation (M), deamidation $(\mathrm{N}, \mathrm{Q})$, and phosphorylation (STY) were set as variable modifications where appropriate. The data was searched against porcine protein sequences using the UPR_Sus scrofa fasta file (as of August 2019), along with the corresponding decoy reverse database. Only unique and razor peptides were used for quantitation.

\section{Statistical analysis}

TMT 10plex quantitation and data analysis was performed in Perseus software (v1.6.0.2) which filtered out contaminant and false positive identifications (decoys) [25]. The data were $\log _{2}$ transformed, and replicates were grouped into RIC and non-RIC. Missing values were imputed from the normal distribution and all samples were normalized by median subtraction. Hierarchical cluster analysis was performed on all the proteins identified by LC-MS/MS and visualized as heat maps using the Pearson correlation algorithm. Volcano plots were generated by applying parametric Student's t-tests between RIC and non-RIC controls. A Christmas tree plot was generated for the phosphoproteomics data where only one replicate was acquired. The mass spectrometry proteomics data have been deposited to the ProteomeXchange Consortium via the PRIDE [26] partner repository with the dataset identifier PXD025273. Gene Ontology and biological pathway enrichment analysis was performed using STRING protein-protein interaction networks \& functional enrichment analysis (https://string-db.org/).

\section{Results and discussion}

We first performed RNA sequencing on renal cortex and medulla tissues of kidney grafts to quantify changes at the transcriptome level (Fig. 1). A total of 19,220 transcripts were successfully quantified across RIC and non-RIC groups (Additional file 2: Tables S1 and S2). In total, 33 transcripts had at least a two-fold change in abundance between groups, at a $p$-value of $\leq 0.05$ (Fig. 2, Additional file 2: Table S3). The most notable of these included heat shock protein beta-1 (HSPB1), monocarboxylate transporter 4 (SLC16A3), C-X-C motif chemokine (CXCL13), $\mathrm{C}-\mathrm{C}$ motif chemokine 2 (CCL2), interleukin-1 receptor type 2 (IL1R2), interleukin-1 beta (IL1B), leukotriene B4 receptor 1 (LTB4R), $\mathrm{Na}(+) / \mathrm{H}(+)$ exchange regulatory cofactor NHE-RF4 (PDZD3), and Ras-like protein family member 10A (RASL10A), all of which were significantly down-regulated following RIC. Moreover, biological pathway analysis revealed a reduction in the transcripts of genes associated with regulation of inflammation following RIC as compared to their non-RIC counterparts (Fig. 2, Additional file 1: Figure S1). Transcript changes were confirmed by performing qPCR on a selected panel of targets which were observed to be dysregulated in RIC in either our dataset, or as previously described in literature. We measured five genes in total in a pooled sample of each group, including IL1B, LTB4R, PDZD3, SLC16A3, and RASL10A (Additional file 2: Table S4). We observed a slight increase in RIC induced SLC16A3 transcripts, but overall, none of the genes quantified reached statistically significance between RIC and non-RIC, with all having a $p$-value of $>0.2$ (Fig. 3).

Following the analysis of the kidney donor biopsies at the transcriptome level, we extended the study to measure changes between the kidney tissue proteomes of the respective RIC and non-RIC groups. Our quantitative proteomic strategy (Fig. 1) successfully quantified a total of 7546 proteins across all samples (Additional file 2: Table S5). Hierarchical clustering analysis revealed no clear discrimination between RIC and non-RIC groups at the proteome level (Fig. 2A). Similarly, and in agreement with the transcriptomics results, the majority of 

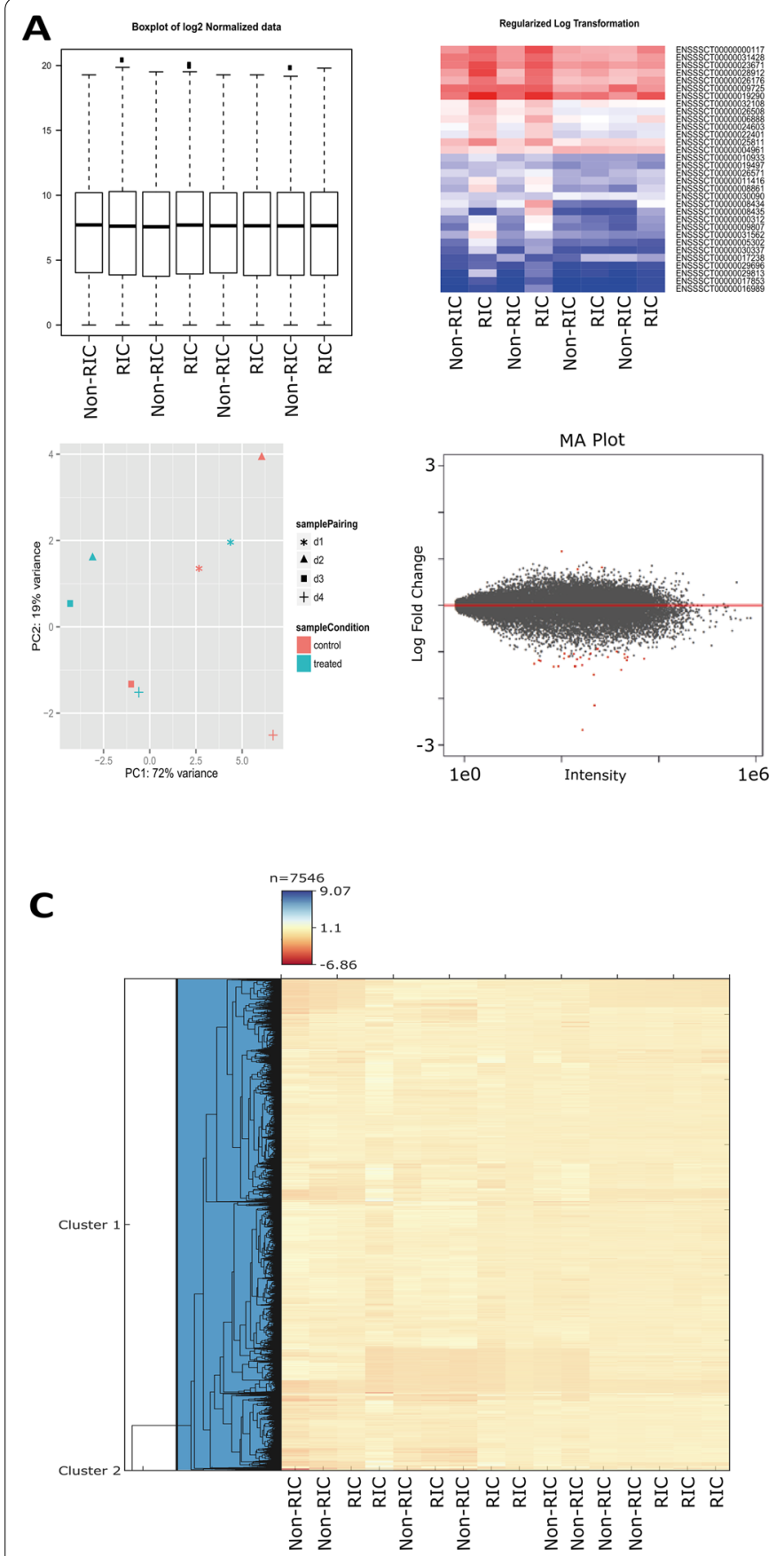
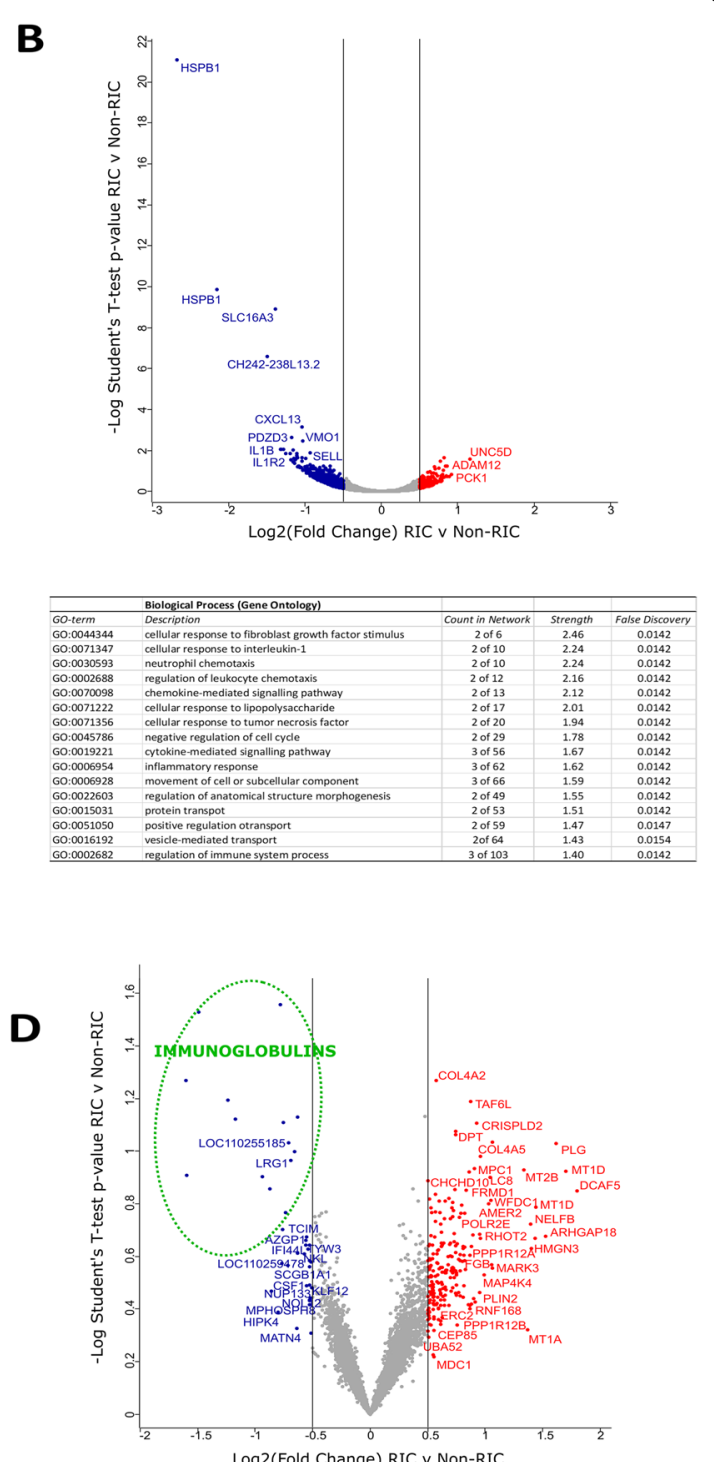

Log2(Fold Change) RIC v Non-RIC

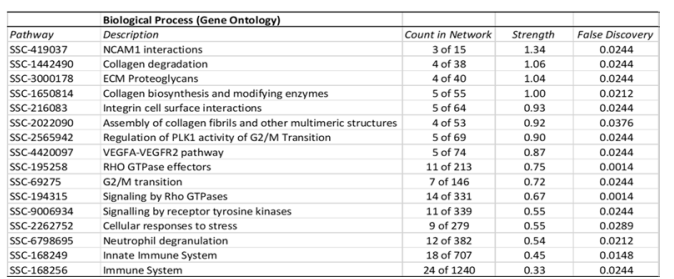

Fig. 2 Transcriptome and proteome changes by RIC. A The top left-hand corner displays Box plots of $\log _{2}$ normalized transcriptomics data. The heat map displays the regularized log-transformed data for the top differentially expressed transcripts. The PCA plot visualizes the regularized log-transformed data which minimizes differences between samples for rows with small counts, and which normalizes with respect to library size. Finally, the MA plot shows the $\log _{2}$ fold changes attributable to a given variable over the mean of normalized counts. Red points have adjusted p-value less than 0.05. B Volcano plot displaying differential transcriptome expression. The $x$-axis measures expression difference by $\log _{2}(f \circ l d$ change), and the $y$-axis indicates statistical significance by - $\log _{10}(p$-value). Down-regulated species are coloured blue, while their up-regulated counterparts are coloured red. Enriched biological processes (Gene Ontology) determined from differentially regulated transcripts (displayed by their gene symbol) are listed underneath the plot. C Hierarchical clustering analysis of RIC and non-RIC proteomes. D Volcano plot displaying differential proteome expression. Down-regulated immunoglobulins of interest are circled in green 


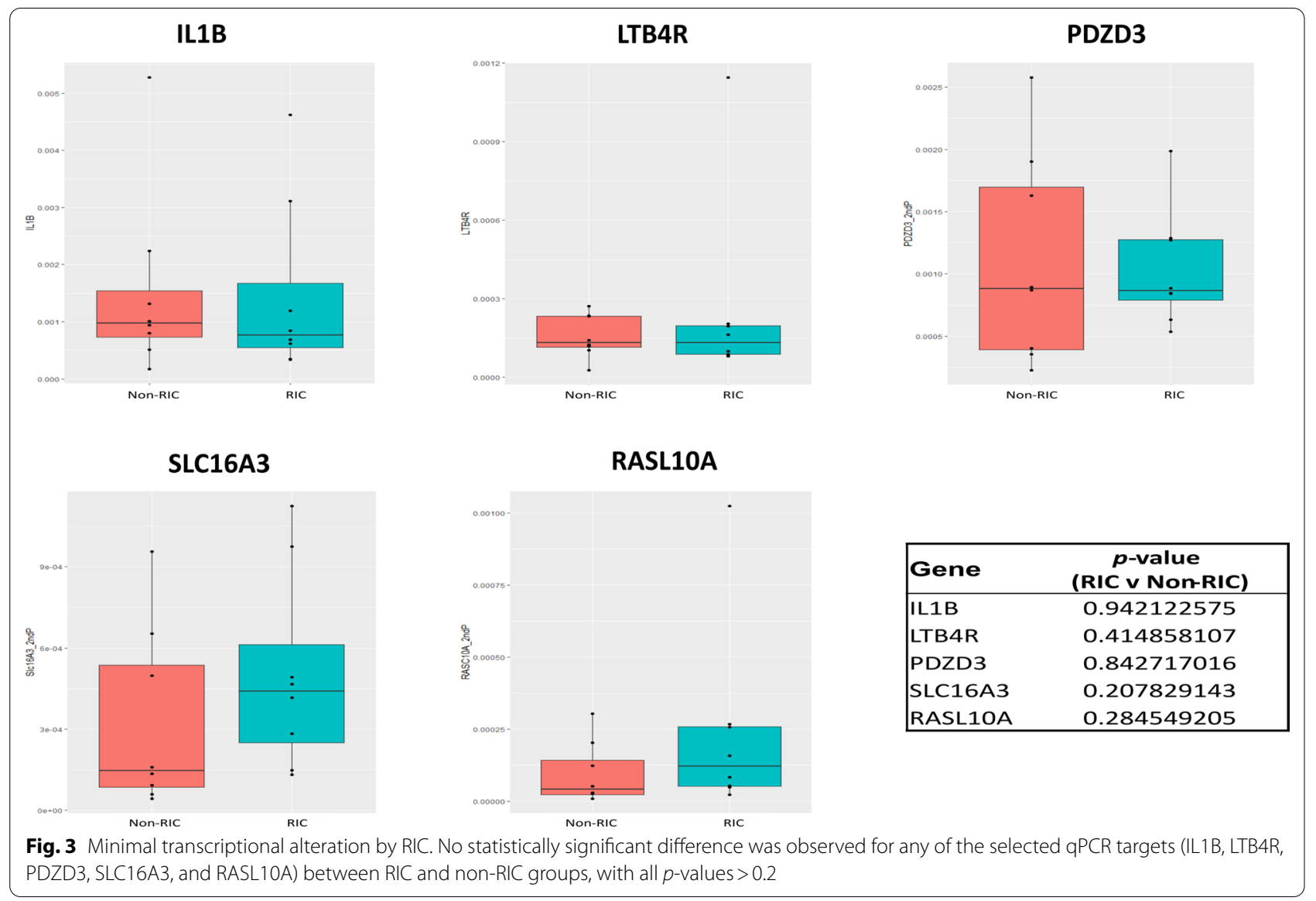

proteins were found to be unchanged between groups. To determine if we could identify trends in the data, we took those proteins with the largest $\left(\geq \log _{2} 0.5\right)$, albeit non-significant, fold changes forward for further analysis. In this context, 252 proteins were deemed to be differentially expressed between RIC and non-RIC groups (Additional file 2: Table S6). Mirroring the transcriptomics results, a dampening of immune response proteins was observed in RIC versus non-RIC tissue, whereby the largest fold changes were observed in approximately 15 Ig-like domain-containing proteins (highlighted in green in Fig. 2B). Attenuation of proteins involved in the inflammatory response associated with IRI could be one of the primary beneficial factors that could contribute to RIC renoprotection. This dampening may serve as a natural defense mechanism against the destruction caused by IRI. Despite this, the inflammatory response generally associated with renal transplantation, in the form of apoptotic cell death, macrophage and neutrophil infiltration, and anti-inflammatory cytokine production, was found to be unaffected by RIC in the pigs studied [27]. We performed pathway enrichment analysis on the differentially expressed proteins. Unfortunately, the Ig-like species described above could not be included due to lacking a mature porcine gene entry (Additional file 1: Figure S2). Mirroring the human CONTEXT study [19], there was an accumulation of muscle-derived and extracellular matrix assembly proteins in the renal tissue following RIC, including the collagens COL1A2, COL2A1, COL4A2, COL4A, COL6A3, fibrillin and fibulin (Fig. 2, Additional file 1: Figure S2), although no tourniquet on a limb but rather clamping of the aorta was used in the present porcine study. Additionally, upregulation of factors associated with innate immunity and signalling, such as lysosome-associated membrane glycoprotein 1 (LAMP1), neutrophil cytosol factor 2 (NCF2), leucine-rich alpha2-glycoprotein (LRG1), myeloperoxidase (MPO), metallothioneins (MT1A, MT1D, and MT2B0 and properdin (CFP) were observed, suggesting the technique provoked the activation of tissue remodeling cascades within the organ itself. Upregulation of these factors provide a favorable environment for wound-healing and repair, and collagen scaffolds have been shown to enhance survival of transplanted cardiomyoblasts and improve function in ischemic rat hearts [28]. Increased collagen production has also been associated with a response to the hypoxic 
or anoxic conditions inherent to surgery, ultimately leading to adhesion development [29]. It is, however, unclear if these proteins provide a protective mechanism in RIC or are just the consequence of tissue damage which could lead to fibrosis. Furthermore, we identified an up-regulation of several components of the blood coagulation cascade, including Vitamin K-dependent protein C (PROC), Fibrinogen beta chain (FGB), and Plasminogen (PLG).

We observed RIC enrichment of Perilipin 2 (PLIN2) which is associated with lipid droplets and has been implicated in IRI of liver tissues [30] and in discriminating renal neoplasms from healthy tissue [31, 32]. PLIN2 may also activate PPARy signaling linked to podocyte apoptosis [33]. RIC may also trigger pathology-linked events, such as a slight increase in proteins associated with mitogen-activated protein kinase [MAPK; specifically, Mitogen-activated protein kinase kinase kinase kinase-4 (MAP4K4)] and protein phosphatase 1 signaling pathways (Fig. 2), similar to spinal cord ischemia/reperfusion injury and in failing human hearts and rodent models of cardiac cell death including IRI [34]. Cysteinerich secretory protein LCCL domain-containing 2 (CRISPLD2), linked to stage-specific diabetes-associated chronic kidney disease [35], was also up-regulated in RIC samples. Similar to the increase in expression in RIC versus non-RIC observed herein, proteomic profiling of the urine of patients with nephrotic syndrome revealed that the kidney-derived Ubiquitin-60S ribosomal protein L40 (UBA52) increased in expression in diseased samples, compared to healthy controls [36].

Interestingly, SLC16A3, IL1B, LTB4R, RASL10A, and CXCL13, which were found to have some of the most prominent changes at the transcript level, were not detected at the proteome level. Furthermore, HSPB1, LTB4R, and PDZD3 transcripts were differentially abundant, but found to be unchanged between non-RIC and RIC groups at the protein level.

To gain more insight into the signaling dynamics underlying these molecular changes in kidney tissue, we performed phosphoproteomics to compare a global pool of all eight RIC samples to that of the eight non-RIC controls. We identified 3524 phosphosites across both groups, from a total of 3626 proteins (Additional file 2: Table S7). No substantial differences were identified in the phosphoproteome between RIC and non-RIC groups, indicating little steady-state effects of RIC on kidney tissue at the indicated time point (Additional file 1: Figure S3).

To determine if a joint analysis could reveal useful insights that could not be deciphered from individual analysis alone, we integrated the transcriptomic and proteomic datasets (Fig. 4A). Once again, the Ig-like proteins that were found to be down-regulated in RIC in the proteomics dataset alone (Fig. 2B) could not be included in this type of analysis due to a lack of matching cognate sequences at the transcriptome level. A low correlation was observed between the two datasets, with a Pearson correlation coefficient of 0.017. We subsequently performed 2D Annotation Enrichment Analysis in an effort to identify pathways which were simultaneously up- or down-regulated in both datasets [37]. Up-regulated biochemical pathways in RIC versus non-RIC and common to both datasets included muscle tissue development/remodeling, extracellular matrix disassembly, ubiquitin-specific protease activity, and steroid hormone receptor activity (Fig. 4). Conversely,
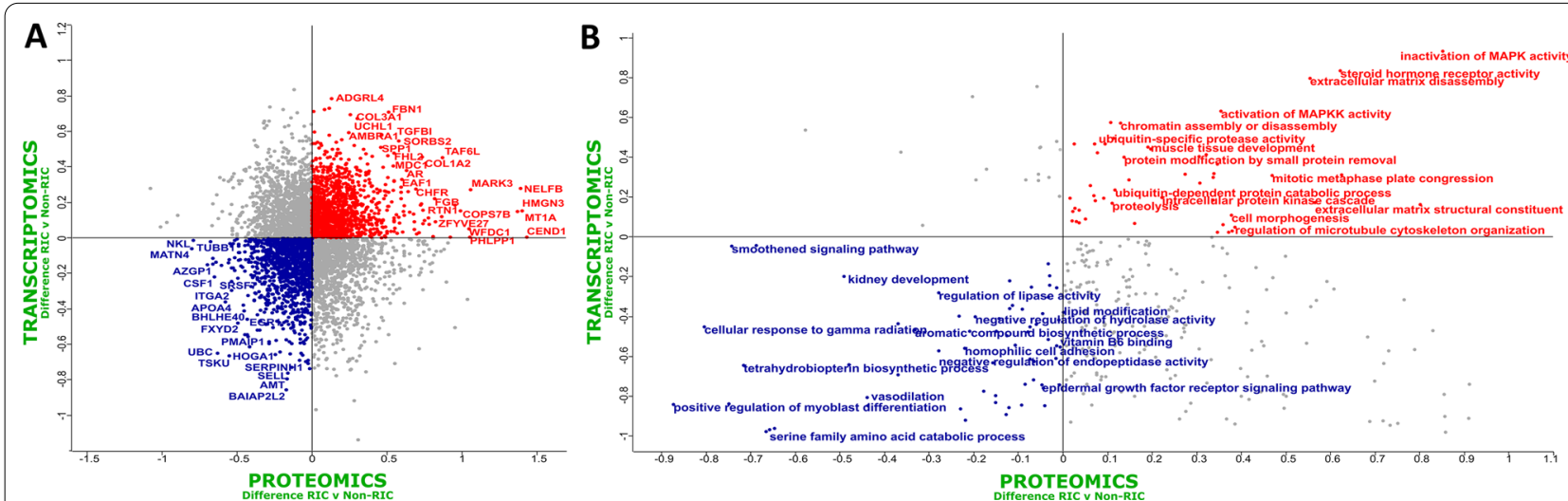

Fig. 4 Integrated Omics reveals RIC induced tissue leakage and reduced inflammation. A Scatter plot of the $\log _{2}$-fold-change between RIC and non-RIC groups of the proteomics on the $x$-axis, versus the transcriptomics on the $y$-axis. A threshold $p$-value of 0.05 was used to determine significantly enriched pathways (displayed). Species which are down-regulated in both datasets are coloured blue, while their up-regulated counterparts are coloured red. A low Pearson correlation coefficient of 0.017 was observed. B 2D Annotation Enrichment Analysis generated using transcripts and proteins which were commonly dysregulated across the two datasets 
down-regulated pathways in RIC versus non-RIC and common to both included kidney development, vasodilation, epidermal growth factor receptor signaling and the regulation of myoblast differentiation.

A possible explanation for the absence of substantial and significant proteomic and transcriptomic changes may be attributed to the condition of kidney tissue used in this study. The tissue had been exposed to multiple insults, including brain death, periods of cold and warm ischemia and reperfusion. This makes it increasingly difficult to fully dissect the (perhaps comparatively minor) effects of RIC, despite a more aggressive RIC regimen than that used in the human setting. A limiting factor of this study concerning Omics profiling is the number of available samples, and the timepoint at which the analyzed biopsies were taken after a 10-h observation phase. This may be too early of a time point to measure a significant change in protein expression, which is possibly reflected in the lack of change identified at the proteomic depth achieved in this study, although the transcriptome profile would not support this notion. Ideally, there would be an observation phase of several days with multiple biopsies. Finally, with the disappointing clinical effects of RIC, the positive effects of RIC regarding GFR and graft reperfusion in the related pig study could reflect a type 1 error or generally reflect a subtle phenomenon observed in this animal model [18].

\section{Conclusions}

In summary, RIC of the pig recipient before kidney graft reperfusion, similar to what was observed in human counterparts, did not result in substantial molecular changes/perturbations in the graft compared to nonRIC controls. The data did suggest, however, that the administered RIC caused a potentially detrimental accumulation of muscle proteins in the kidney graft tissue and also triggered a subtle interplay between the innate and adaptive immune response, with a dampening of the latter. Ultimately, however, our discovery studies showed no major differences in the transcriptomes and proteomes of porcine kidney transplant tissue following RIC, despite excellent depth of coverage in each. Our results, therefore, support the conclusion that the RIC protocol used does not substantially protect against ischemia/reperfusion injury.

\section{Abbreviations}

AKI: Acute kidney injury; ATP: Adenosine triphosphate; GFR: Glomerular filtration rate; IRI: Ischemic/reperfusion injury; RIC: Remote ischemic preconditioning; TMT:Tandem mass tag.

\section{Supplementary Information}

The online version contains supplementary material available at https://doi. org/10.1186/s12014-022-09343-3.

Additional file 1: Figure S1. Transcriptomic pathway enrichment analysis reveals subtle alteration of tissue inflammation by RIC. All transcripts ( $n=33$ ) which were found to be dysregulated in RIC versus non-RIC controls were searched against the Sus scrofa database in STRING. Only interactions of the highest confidence (scores $>0.90$ ) were included in the analysis. Genes associated with immune regulation are highlighted, with those linked to interleukin biology coloured red, while cytokines are coloured purple. Figure S2. Proteomic pathway enrichment analysis uncovers RIC induced tissue leakage and altered inflammation. Proteins ( $n=252$ ) which were found to have the greatest dysregulation in RIC versus non-RIC controls were searched against the Sus scrofa database in STRING. Only interactions of the highest confidence (scores $>0.90$ ) were included in the analysis. Proteins/genes of interest are highlighted, with proteins associated with muscle and ECM coloured in blue, blood coagulation in green, and factors associated with innate immunity coloured red. Figure S3. Kidney tissue phosphoproteomics unaffected by RIC. A) Abundance plots indicate no significant difference in the phosphoproteome between RIC and control groups. B) A Christmas tree plot, with Significance B thresholds colour coded. Minor changes were observed between groups.

Additional file 2: Table S1. Transcriptome sequence alignments. An average sequence alignment of $82 \%$ was achieved across all samples. Table S2. List of transcripts identified by transcriptomics. A total of 19,220 transcripts were successfully identified across groups. Table S3. Transcripts differentially expressed between RIC and Non-RIC groups. Table S4. qPCR validation on a panel of targets from discovery transcriptomics. IL1B, LTB4R, PDZD3, SLC16A3, and RASL10A were quantified by qPCR. We observed a slight increase in RIC induced SLC16A3 transcripts, but overall, none of the genes quantified reached statistically significance between RIC and non-RIC, with all having a $p$-value of $>0.2$. Table S5. List of proteins identified by proteomics. A total of 7,546 proteins were successfully identified across groups. Table S6. Proteins differentially expressed between RIC and Non-RIC groups. Table S7. List of proteins and phosphosites identified by phosphoproteomics. A total of 3,524 phosphosites were successfully identified across groups.

\section{Acknowledgements}

We thank Raphael Heilig and Roman Fischer, head of the Discovery Proteomics Facility, for expert help with mass spectrometry analysis. The authors would also like to thank members of the Kessler, Jespersen, and Ploeg research groups for helpful discussions and technical support.

\section{Authors' contributions}

$\mathrm{DOB}, \mathrm{AT}, \mathrm{HH}, \mathrm{EP}, \mathrm{XY}, \mathrm{PS}, \mathrm{KR}$ and NS performed the experiments. BJ, RJP and BMK conceptualized the study. DOB, AT, BJ, RJP and BMK wrote the manuscript. DOB, AT, HH, EP, PS, KR and NS analyzed the data. All authors read and approved the final manuscript.

\section{Funding}

Funding was provided by the Novo Nordisk Foundation, The Danish Medical Research Council, The Danish Kidney Association (Nyreforeningen), Department of Clinical Medicine, Aarhus University, Nuffield Department of Medicine, University of Oxford, and Nuffield Department of Surgical Sciences, University of Oxford.

\section{Availability of data and materials}

The mass spectrometry raw data files have been deposited under PRIDE Submission PXD025273. The transcriptomics dataset was deposited in ArrayExpress (AE) at EBI under the accession number E-MTAB-10872. 


\section{Declarations}

Ethics approval and consent to participate

Kidney graft tissue samples were taken from the porcine RIC study [18].

\section{Consent for publication}

All authors have read the manuscript and given their consent for publication.

\section{Competing interests}

The authors have no conflicts of interest to declare.

\section{Author details}

${ }^{1}$ Target Discovery Institute, Centre for Medicines Discovery, Nuffield Department of Medicine, University of Oxford, Oxford, UK. ${ }^{2}$ Nuffield Department of Surgical Sciences and Oxford Biomedical Research Centre, University of Oxford, Oxford, UK. ${ }^{3}$ Sir William Dunn School of Pathology, University of Oxford, Oxford, UK. ${ }^{4}$ Department of Renal Medicine, Aarhus University Hospital, Aarhus, Denmark. ${ }^{5}$ Department of Anaesthesiology, Aalborg University Hospital, Aalborg, Denmark. ${ }^{6}$ Department of Clinical Medicine, Aarhus University, Aarhus, Denmark. ${ }^{7}$ Department of Anaesthesiology and Intensive Care Medicine, Aarhus University Hospital, Aarhus, Denmark.

Received: 31 August 2021 Accepted: 3 February 2022

Published online: 14 February 2022

\section{References}

1. Wu MY, et al. Current mechanistic concepts in ischemia and reperfusion injury. Cell Physiol Biochem. 2018;46(4):1650-67.

2. Zhao $\mathrm{H}$, et al. Ischemia-reperfusion injury reduces long term renal graft survival: mechanism and beyond. EBioMedicine. 2018;28:31-42.

3. Sharfuddin AA, Molitoris BA. Pathophysiology of ischemic acute kidney injury. Nat Rev Nephrol. 2011;7(4):189-200.

4. Zhou D, et al. Remote ischemic conditioning: a promising therapeutic intervention for multi-organ protection. Aging. 2018;10(8):1825-55.

5. Thuret $R$, et al. Ischemic pre- and post-conditioning: current clinical applications. Prog Urol. 2014;24(Suppl 1):S56-61.

6. Jiang $T$, et al. The role of remote ischemic preconditioning in ischemiareperfusion injury in rabbits with transplanted lung. Clin Lab. 2015;61(5-6):481-6.

7. Vasdekis $\mathrm{SN}$, et al. The role of remote ischemic preconditioning in the treatment of atherosclerotic diseases. Brain Behav. 2013:3(6):606-16.

8. Vrakas $\mathrm{G}$, et al. Synergistic effect of ischemic preconditioning and antithrombin in ischemia-reperfusion injury. Exp Clin Transplant. 2017:15(3):320-8.

9. Magyar Z, et al. Beneficial effects of remote organ ischemic preconditioning on micro-rheological parameters during liver ischemia-reperfusion in the rat. Clin Hemorheol Microcirc. 2018;70(2):181-90.

10. Saeki I, et al. Ischemic preconditioning and remote ischemic preconditioning have protective effect against cold ischemia-reperfusion injury of rat small intestine. Pediatr Surg Int. 2011;27(8):857-62.

11. Qi B, et al. Effect of remote ischemic preconditioning among donors and recipients following pediatric liver transplantation: a randomized clinical trial. World J Gastroenterol. 2021;27(4):345-57.

12. Roubille $F$, et al. Effects of remote ischemic conditioning on kidney injury in at-risk patients undergoing elective coronary angiography (PREPARE study): a multicenter, randomized clinical trial. Sci Rep. 2019;9(1):11985.

13. Wu G, et al. Effect of remote ischemic preconditioning on hepatic ischemia-reperfusion injury in patients undergoing liver resection: a randomized controlled trial. Minerva Anestesiol. 2020;86(3):252-60.

14. Tuncer FB, et al. Ischemic preconditioning and iloprost reduces ischemiareperfusion injury in jejunal flaps: an animal model. Plast Reconstr Surg. 2019;144(1):124-33.

15. Jung KW, et al. Effect of remote ischemic preconditioning conducted in living liver donors on postoperative liver function in donors and recipients following liver transplantation: a randomized clinical trial. Ann Surg. 2020:271(4):646-53.
16. Fuller TF, et al. Ischemic preconditioning improves rat kidney graft function after severe ischemia/reperfusion injury. Transplant Proc. 2005:37(1):377-8.

17. Krogstrup NV, et al. Remote ischemic conditioning on recipients of deceased renal transplants does not improve early graft function: a multicenter randomized, controlled clinical trial. Am J Transplant. 2017;17(4):1042-9.

18. Soendergaard P, et al. Improved GFR and renal plasma perfusion following remote ischaemic conditioning in a porcine kidney transplantation model. Transpl Int. 2012;25(9):1002-12.

19. Thorne AM, et al. Subclinical effects of remote ischaemic conditioning in human kidney transplants revealed by quantitative proteomics. Clin Proteomics. 2020;17(1):39.

20. Nielsen MB, et al. Remote ischaemic conditioning and early changes in plasma creatinine as markers of one year kidney graft function-a followup of the CONTEXT study. PLOS ONE. 2019;14(12):e0226882.

21. Barklin A, et al. Does brain death induce a pro-inflammatory response at the organ level in a porcine model? Acta Anaesthesiol Scand. 2008;52(5):621-7

22. Athar A, et al. ArrayExpress update-from bulk to single-cell expression data. Nucleic Acids Res. 2019;47(D1):D711-d715.

23. $\mathrm{Li} \mathrm{Q}$, et al. Evaluation of potential reference genes for relative quantification by RT-qPCR in different porcine tissues derived from feeding studies. Int J Mol Sci. 2011;12(3):1727-34.

24. Dullaert-de Boer $M$, et al. Variability and cost implications of three generations of the Roche LightCycler ${ }^{\circledR}$ 480. PLoS ONE. 2018;13(1):e0190847.

25. Tyanova S, et al. The Perseus computational platform for comprehensive analysis of (prote)omics data. Nat Methods. 2016;13(9):731-40.

26. Perez-Riverol Y, et al. The PRIDE database and related tools and resources in 2019: improving support for quantification data. Nucleic Acids Res. 2019:47(D1):D442-d450.

27. Secher $\mathrm{N}$, et al. No effect of remote ischaemic conditioning on inflammation in a porcine kidney transplantation model. Transpl Immunol. 2014;31(2):98-104.

28. Kutschka l, et al. Collagen matrices enhance survival of transplanted cardiomyoblasts and contribute to functional improvement of ischemic rat hearts. Circulation. 2006;114(1 Suppl):1167-73.

29. Saed GM, Diamond MP. Hypoxia-induced irreversible up-regulation of type I collagen and transforming growth factor-beta1 in human peritoneal fibroblasts. Fertil Steril. 2002;78(1):144-7.

30. Huang $S$, et al. Comprehensive and combined omics analysis reveals factors of ischemia-reperfusion injury in liver transplantation. Epigenomics. 2019;11(5):527-42.

31. Xu S, et al. Perilipin 2 impacts acute kidney injury via regulation of PPARa. J Immunol Res. 2021;2021:9972704.

32. Song $Y$, et al. Data-independent acquisition-based quantitative proteomic analysis reveals potential biomarkers of kidney cancer. Proteomics Clin Appl. 2017. https://doi.org/10.1002/prca.201700066.

33. Dai ZW, et al. Perilipin2 inhibits diabetic nephropathy-induced podocyte apoptosis by activating the PPARY signaling pathway. Mol Cell Probes. 2020;53:101584.

34. Te Lintel Hekkert M, et al. Preclinical trial of a MAP4K4 inhibitor to reduce infarct size in the pig: does cardioprotection in human stem cellderived myocytes predict success in large mammals? Basic Res Cardiol. 2021:116(1):34.

35. Lecamwasam A, et al. DNA methylation profiling identifies epigenetic differences between early versus late stages of diabetic chronic kidney disease. Nephrol Dial Transplant. 2021;36(11):2027-38.

36. Wang $Y$, et al. Proteomic profile-based screening of potential protein biomarkers in the urine of patients with nephrotic syndrome. Mol Med Rep. 2017;16(5):6276-84.

37. Cox J, Mann M. 1D and 2D annotation enrichment: a statistical method integrating quantitative proteomics with complementary high-throughput data. BMC Bioinformatics. 2012:13(Suppl 16):S12.

\section{Publisher's Note}

Springer Nature remains neutral with regard to jurisdictional claims in published maps and institutional affiliations. 